\title{
Modeling of viscoelastic shear: A nonlinear stick-slip formulation
}

\author{
H.T. Banks and Negash G. Medhin \\ Center for Research in Scientific Computation \\ North Carolina State University \\ Raleigh, NC 27695-8205 \\ Gabriella A. Pinter \\ Department of Mathematical Sciences \\ University of Wisconsin, Milwaukee \\ Milwaukee, WI 53201-0413
}

February 20, 2006

\begin{abstract}
We present a class of nonlinear dynamic viscoelastic models for materials subjected to shear stress. The model equations are based on a continuum variation of a reptation model in which chemically cross-linked (CC) systems of molecules act as constraint boxes per unit volume for physically constrained (PC) systems of molecules. Results from validating the model with dynamic shear experiments are given and a stability analysis for the corresponding linearized systems is discussed.
\end{abstract}

\section{Introduction}

In this paper we derive for the first time a continuum model combined with molecular based internal dynamics for shear deformations in viscoelastic materials. The resulting partial differential equation model is coupled to ordinary differential equations for internal strains via a nonlinear stick-slip molecular theory. An initial step toward validating the models with carefully designed experiments is discussed.

Various molecular and phenomenological models have been proposed to model rubber deformations. A more scientifically satisfactory model can be obtained by combining molecular and continuum mechanics approaches. The fundamental ideas originate in the presentations of Doi and Edwards [8] and Johnson and Stacer [11] as extended in [4, 5]. To make the presentation in this paper self contained we first briefly summarize some of the background material presented in $[3,4,5]$.

A dynamic model for rubber viscoelasticity has been proposed in [11] based on a continuum simulation of the reptation model considered in [8]. In step-strain relaxations of polymers with constraint (stick-slip) theory, PC-molecules deform with CC-molecules, contract and creep to return to a lower energy, higher entropy state. As a result, the total energy density at a constant strain dissipates in time and viscoelastic behavior results.

Such a linear continuum model of rubber viscoelasticity is developed in [11] by considering the CC-linked molecules as providing cells or boxes with entrapped molecular segments and placing a unit cell or box at each point of the rubber continuum. This approach was extended 
to nonlinear models for rubber undergoing tensile deformations in [4, 5]. Here we consider the case where the CC-box experiences a shear stress as a result of which the PC-molecules undergo a parallel deformation. In our formulation the PC-molecules act as internal variables in the relaxation of the CC-box.

\section{Continuum model of Johnson and Stacer}

When the Johnson-Stacer [11] CC-system experiences shear deformations, the PC-system undergoes deformation along with it. The relaxation of the PC-system is determined by the history of the CC-system along with thermodynamic considerations. On the other hand the PC-system deformation acts as an internal variable affecting the relaxation of the CCsystem. The tendency for the PC-molecule to return to a lower energy and higher entropy configuration forms the basis of the viscoelastic model in the deformation process. A PCmolecule entrapped in a CC-molecular box undergoes a deformation in the direction of the shear when the CC-box experiences shear. After the initial induced stress, the PC-molecule relaxes even if the CC-box remains deformed. Let the CC-constraint tube have length $L(t)$ and the representative entrapped PC-molecule have length $\ell(t)$. The model in [11] assumes that the length of the CC-tube is approximated as a step function of time and $\ell(0)=L(0)$. Suppose instantaneous step-strain deformation of the CC-system results in the PC-molecule having length $\ell^{*}$. A model of the PC-molecule returning to its original contour length is given by

$$
\ell(t)=\ell(0)+\left[\ell^{*}-\ell(0)\right] e^{-t / \tau}
$$

If the initial stretch for the CC-box is $\Delta L_{0}$, relating the stretches in the form $\frac{\Delta \ell_{0}}{\Delta L_{0}}=\frac{\ell_{0}}{L_{0}}$ leads to the relation

$$
\Delta \ell_{0}=\left(\frac{\ell_{0}}{L_{0}}\right) \Delta L_{0} .
$$

Then in the time interval $t_{0}<t<t_{1}$ we have

$$
\ell(t)=\ell_{0}+\frac{\ell_{0}}{L_{0}} \Delta L_{0} e^{-\left(t-t_{0}\right) / \tau}
$$

where $\tau$ is the relaxation time for the slip motion of the PC-molecule.

Setting $t=t_{1}$, we have

$$
\begin{aligned}
\ell\left(t_{1}\right) & =\ell_{0}+\frac{\ell_{0}}{L_{0}} \Delta L_{0} e^{-\left(t_{1}-t_{0}\right) / \tau} \\
L_{1} & =L_{0}+\Delta L_{0} .
\end{aligned}
$$

Next we suppose that the CC-box is subjected to an instantaneous shear step deformation $\Delta L_{1}$ at $t=t_{1}$. For $t$ in the time interval $\left(t_{1}, t_{2}\right)$ we have

$$
\begin{aligned}
\ell(t) & =\ell_{0}+\left[\left(\ell_{1}+\Delta \ell_{1}\right)-\ell_{0}\right] e^{-\left(t-t_{1}\right) / \tau} \\
& =\ell_{0}+\frac{\ell_{0}}{L_{0}} \Delta L_{0} e^{-\left(t-t_{0}\right) / \tau}+\frac{\ell_{1}}{L_{1}} \Delta L_{1} e^{-\left(t-t_{1}\right) / \tau} .
\end{aligned}
$$


Similarly, after another instantaneous shear step deformation $\Delta L_{2}$ at $t=t_{2}$, for $t_{2}<t<t_{3}$ we have

$$
\begin{aligned}
\ell(t) & =\ell_{0}+\frac{\ell_{0}}{L_{0}} \Delta L_{0} e^{-\left(t-t_{0}\right) / \tau}+\frac{\ell_{1}}{L_{1}} \Delta L_{1} e^{-\left(t-t_{1}\right) / \tau} \\
& +\frac{\ell_{2}}{L_{2}} \Delta L_{2} e^{-\left(t-t_{2}\right) / \tau} .
\end{aligned}
$$

Following [11] and taking the limit, as $\Delta t_{i} \rightarrow 0$, of a succession of such step strains, we are led to the formula

$$
\ell(t)=\ell_{0}+\int_{0}^{t} \frac{\ell(s)}{L(s)} \frac{d L(s)}{d s} e^{-(t-s) / \tau} d s
$$

for the length of the PC-molecule.

We make the following observation for the linear theory that will be subsequently modified for a nonlinear theory. If $\Delta \ell_{i}$ and $\Delta L_{i}$ represent instantaneous step-stretches at $t=t_{i}$, we have assumed the fundamental relationship

$$
\frac{\Delta \ell_{i}}{\Delta L_{i}}=\frac{\ell_{i}}{L_{i}}
$$

where $\ell_{i}=\ell\left(t_{i}\right), L_{i}=L\left(t_{i}\right)$.

\section{Shear deformation in the CC-box and deformation of the PC-molecule}

A deformation that is close to the axial extension and to which we can readily apply the continuum ideas of Johnson and Stacer is generalized simple shear as depicted in Figure 1. We suppose that the unit CC-box undergoes a deformation of the type $x \rightarrow x+u(y), y \rightarrow y$, $z \rightarrow z$.

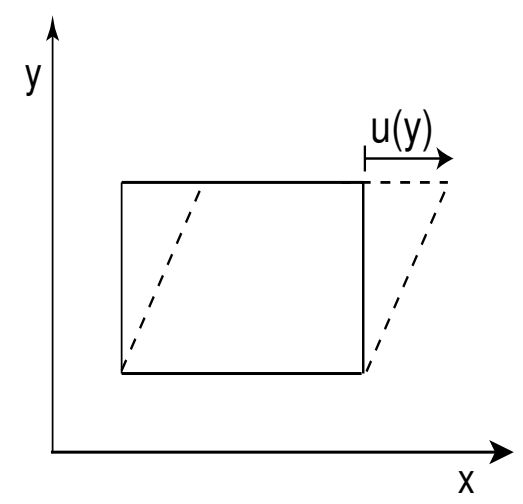

Figure 1: Simple shear. 
The configuration gradient $[1,2]$ for this deformation is given by

$$
A=\left(\begin{array}{ccc}
1 & u^{\prime}(y) & 0 \\
0 & 1 & 0 \\
0 & 0 & 1
\end{array}\right)
$$

The configuration gradient $A$ can be written in a unique way as a product of a stretch tensor $E$ and rotation $R$ as

$$
A=E R,
$$

where $E^{2}=A^{*} A$. The eigenvalues $\xi_{1}, \xi_{2}, \xi_{3}$ of $A^{*} A$ are given by

$$
\begin{aligned}
\xi_{1} & =1+\frac{1}{2}\left[u^{\prime}(y)\right]^{2}+u^{\prime}(y) \sqrt{1+\frac{1}{4}\left[u^{\prime}(y)\right]^{2}} \\
& =1+u^{\prime}(y)+\frac{1}{2}\left[u^{\prime}(y)\right]^{2}+\frac{1}{8}\left[u^{\prime}(y)\right]^{3}-\ldots \\
\xi_{2} & =1+\frac{1}{2}\left[u^{\prime}(y)\right]^{2}-u^{\prime}(y) \sqrt{1+\frac{1}{4}\left[u^{\prime}(y)\right]^{2}} \\
& =1-u^{\prime}(y)+\frac{1}{2}\left[u^{\prime}(y)\right]^{2}-\frac{1}{8}\left[u^{\prime}(y)\right]^{3}+\ldots \\
\xi_{3} & =1 .
\end{aligned}
$$

We note that $\xi_{1} \xi_{2}=1$ and thus also $\xi_{1} \xi_{2} \xi_{3}=1$. For small deformations we set

$$
\begin{aligned}
& \lambda_{1 c}=\sqrt{\xi_{1}}=1+\frac{1}{2} u^{\prime}(y)+\frac{1}{4}\left[u^{\prime}(y)\right]^{2}+\frac{1}{16}\left[u^{\prime}(y)\right]^{3} \mp \ldots \\
& \lambda_{2 c}=\sqrt{\xi_{2}}=1-\frac{1}{2} u^{\prime}(y)+\frac{1}{4}\left[u^{\prime}(y)\right]^{2}-\frac{1}{16}\left[u^{\prime}(y)\right]^{3}+\ldots \\
& \lambda_{3 c}=1 .
\end{aligned}
$$

The quantities $\lambda_{1 c}, \lambda_{2 c}, \lambda_{3 c}$ are the principal stretches for the CC-shear deformations.

Although the CC-box experiences shear deformation, the entrapped PC-molecules experience deformations that can be simple shear, simple elongation, or a combination thereof, depending on the orientation of the $\mathrm{PC}$ molecule in the entanglement with the CC-box. In any case, we assume that the $\mathrm{PC}$ deformation $\delta u_{p}$ is in the same direction as the CC-box shear, and thus the principal stretches for the PC-molecules may be assumed to obey the relationships

$$
\lambda_{2 p}=\lambda_{3 p}=\frac{1}{\sqrt{\lambda_{1 p}}} .
$$

To develop relationships between the principal stretches $\lambda_{i c}$ and $\lambda_{i p}$ we use (2.1) and (2.2). The equation (2.1) suggests that we write

$$
\lambda_{1 p}=1+\int_{0}^{t} \frac{\lambda_{1 p}(s)}{\lambda_{1 c}(s)} \frac{d \lambda_{1 c}(s)}{d s} e^{-(t-s) / \tau} d s .
$$

The equation (2.2) further suggests that we assume the relationship

$$
\frac{\partial \lambda_{i p}}{\partial \lambda_{j c}}=\frac{\lambda_{i p}}{\lambda_{j c}} \delta_{i j}
$$


where $\delta_{i j}$ is the Kronecker delta.

In (3.2) we assume that $u$ also depends on time. Thus,

$$
\begin{aligned}
\lambda_{1 c} & =1+\frac{1}{2} \partial_{y} u(t, y)+\frac{1}{4}\left[\partial_{y} u(t, y)\right]^{2}+\frac{1}{16}\left[\partial_{y} u(t, y)\right]^{3} \mp \ldots \\
\lambda_{2 c} & =1-\frac{1}{2} \partial_{y} u(t, y)+\frac{1}{4}\left[\partial_{y} u(t, y)\right]^{2}-\frac{1}{16}\left[\partial_{y} u(t, y)\right]^{3} \pm \ldots \\
\lambda_{3 c} & =1 .
\end{aligned}
$$

Using (3.6), we obtain from (3.4) the equation

$$
\begin{array}{r}
\lambda_{1 p}=1+\int_{0}^{t} \lambda_{1 p}(s)\left(1-\frac{1}{2} \partial_{y} u(s, y)+\frac{3}{8}\left[\partial_{y} u(s, y)\right]^{3}+\ldots\right) . \\
\left(\frac{1}{2} \partial_{s} \partial_{y} u(s, y)+\frac{1}{2} \partial_{y} u(s, y) \partial_{s} \partial_{y} u(s, y)+\ldots\right) \cdot e^{-(t-s) / \tau} d s .
\end{array}
$$

From (3.7) we find

$$
\lambda_{1 p} \approx 1+\int_{0}^{t} \lambda_{1 p}(s)\left(1-\frac{1}{2} \partial_{y} u(s, y)\right) \cdot \frac{1}{2} \frac{\partial^{2}}{\partial s \partial y} u(s, y) e^{-(t-s) / \tau} d s .
$$

Setting $\lambda_{1 p} \approx 1+\delta u_{p}$ where $\delta u_{p}=\delta u_{p}(t, y)$, we see from (3.8) that

$$
\begin{aligned}
\delta u_{p}(t, y) & \approx \frac{1}{2} \partial_{y} u(t, y)-\frac{1}{2 \tau} \int_{0}^{t} \partial_{y} u(s, y) e^{-(t-s) / \tau} d s \\
& +\int_{0}^{t}\left[\delta u_{p}-\frac{1}{2} \partial_{y} u\right] \frac{1}{2} \partial_{s} \partial_{y} u(s, y) e^{-(t-s) / \tau} d s-\frac{1}{2} \partial_{y} u(0, y) e^{-t / \tau}
\end{aligned}
$$

Next, let (assume that $\partial_{y} u(0, y)=0$ )

$$
w=\delta u_{p}-\frac{1}{2} \partial_{y} u(t, y) .
$$

Then from (3.9)

$$
\partial_{t} w+\left(\frac{1}{\tau}-\frac{1}{2} \frac{\partial^{2} u}{\partial t \partial y}\right) w=-\frac{1}{2 \tau} \partial_{y} u(t, y) .
$$

Thus

$$
\begin{aligned}
\delta u_{p}(t, y) \approx & \frac{1}{2} \partial_{y} u(t, y)-\frac{1}{2 \tau} \int_{0}^{t} e^{-(t-s) / \tau} \partial_{y} u(s, y) d s \\
- & \frac{1}{\tau} \int_{0}^{t} e^{-(t-s) / \tau}\left[\frac{1}{2}\left(\partial_{y} u(t, y)-\partial_{y} u(s, y)\right)+\frac{1}{4}\left(\partial_{y} u(t, y)-\partial_{y} u(s, y)\right)^{2}\right] . \\
& \frac{1}{2} \partial_{y} u(s, y) d s .
\end{aligned}
$$

From this last equation we are persuaded to make the approximation

$$
\delta u_{p}(t, y)=\frac{1}{2} \partial_{y} u(t, y)-\frac{1}{2 \tau} \int_{0}^{t} e^{-(t-s) / \tau} \partial_{y} u(s, y) d s .
$$




\section{The dynamics equation}

We proceed to write the dynamic equation for a particular rubber material considered in [11]. For this particular material the energy density proposed in [11] consists of two parts, $W_{c c}$ and $W_{p c}$, where $W_{c c}$ is the contribution to the energy density of a unit CC-box from the cross-linked molecules constituting the box. A contribution to the energy density function from entrapped PC-molecules is denoted $W_{p c}$. The $W_{c c}$ and $W_{p c}$ proposed in [11] are given by

$$
\begin{aligned}
W_{c c}= & 105\left(\lambda_{1 c}^{2}+\lambda_{2 c}^{2}+\lambda_{3 c}^{2}-3\right)+ \\
& 103\left(\lambda_{1 c}^{2} \lambda_{2 c}^{2}+\lambda_{1 c}^{2} \lambda_{3 c}^{2}+\lambda_{2 c}^{2} \lambda_{3 c}^{2}-3\right) \\
W_{p c}= & 169\left(\lambda_{1 p}^{2}+\lambda_{2 p}^{2}+\lambda_{3 p}^{2}-3\right)+0.0138\left(\lambda_{1 p}^{2}+\lambda_{2 p}^{2}+\lambda_{3 p}^{2}-3\right)^{2}+ \\
& 7.89\left(\lambda_{1 p}^{2} \lambda_{2 p}^{2}+\lambda_{1 p}^{2} \lambda_{3 p}^{2}+\lambda_{2 p}^{2} \lambda_{3 p}^{2}-3\right)^{3}
\end{aligned}
$$

where the specific numbers in these expressions are derived from validation with experimental data of Young and Danik (see [10]). Using the fact $\lambda_{1 c}^{2}=\xi_{1}$ and $\lambda_{2 c}^{2}=\xi_{2}$ and (3.1), we find

$$
W_{c c}=208 u_{y}^{2}+a u_{y}^{3}+\cdots
$$

Thus

$$
\frac{\partial W_{c c}}{\partial u_{y}}=416 u_{y}+a_{2} u_{y}^{2}+a_{3} u_{y}^{3}+\cdots \equiv \hat{g}_{e}\left(u_{y}\right) \approx 416 u_{y} .
$$

Next using (3.3) we have

$$
\begin{aligned}
W_{p c} & =169\left(\lambda_{1 p}^{2}+\frac{2}{\lambda_{1 p}}-3\right)+0.0138\left(\lambda_{1 p}^{2}+\frac{2}{\lambda_{1 p}}-3\right)^{2} \\
& +7.89\left(2 \lambda_{1 p}+\frac{1}{\lambda_{1 p}^{2}}-3\right)^{3}, \\
\frac{\partial W_{p c}}{\partial \lambda_{1 p}} & =169\left(2 \lambda_{1 p}-\frac{2}{\lambda_{1 p}^{2}}\right)+0.0276\left(\lambda_{1 p}^{2}+\frac{2}{\lambda_{1 p}}-3\right)\left(2 \lambda_{1 p}-\frac{2}{\lambda_{1 p}^{2}}\right) \\
& +23.67\left(2 \lambda_{1 p}+\frac{1}{\lambda_{1 p}^{2}}-3\right)^{2}\left(2-\frac{2}{\lambda_{1 p}^{3}}\right) .
\end{aligned}
$$

Recalling $\lambda_{1 p} \approx 1+\delta u_{p}$ we have

$$
\frac{\partial W_{p c}}{\partial \lambda_{1 p}} \approx 1014 \delta u_{p}
$$


Using (3.5) we have

$$
\begin{aligned}
\frac{\partial W_{p c}}{\partial \lambda_{1 c}} & =\frac{\partial W_{p c}}{\partial \lambda_{1 p}} \frac{\partial \lambda_{1 p}}{\partial \lambda_{1 c}}=\frac{\partial W_{p c}}{\partial \lambda_{1 p}} \frac{\lambda_{1 p}}{\lambda_{1 c}} \\
\frac{\lambda_{1 p}}{\lambda_{1 c}} & \approx \frac{1+\delta u_{p}}{\lambda_{1 c}} \approx 1+\delta u_{p}-\frac{1}{2} \partial_{y} u-\frac{1}{2} \delta u_{p} \partial_{y} u+\frac{1}{4}\left(\partial_{y} u\right)^{2}+\ldots \\
\frac{\partial W_{p c}}{\partial u_{y}} & =\frac{\partial W_{p c}}{\partial \lambda_{1 c}} \frac{\partial \lambda_{1 c}}{\partial u_{y}} \approx 1014 \delta u_{p}\left(1+\delta u_{p}-\frac{1}{2} \partial_{y} u-\frac{1}{2} \delta u_{p} \partial_{y} u+\frac{1}{4}\left(\partial_{y} u\right)^{2}+\ldots\right) \frac{1}{2} \\
& =512 \delta u_{p}\left(1+\delta u_{p}-\frac{1}{2} u_{y}-\frac{1}{2} \delta u_{p} \partial_{y} u+\frac{1}{4}\left(\partial_{y} u\right)^{2}+\ldots\right) \\
& \approx 512 \delta u_{p} .
\end{aligned}
$$

Thus to first order we have

$$
\frac{\partial W_{p c}}{\partial u_{y}} \approx 512 \delta u_{p}
$$

Now we consider the shear vibration of a rubber sample with a square cross sectional area $A_{c}$ and density $\rho$. From (4.2) and (3.10)

$$
\frac{\partial W_{p c}}{\partial u_{y}} \approx 256 \partial_{y} u(t, y)-\frac{256}{\tau} \int_{0}^{t} e^{-(t-s) / \tau} \partial_{y} u(s, y) d s
$$

The shear dynamics of the rubber sample are given by

$$
\rho A_{c} \partial_{t}^{2} u-\partial_{y}\left(\frac{\partial W_{c c}}{\partial u_{y}}+\frac{\partial W_{p c}}{\partial u_{y}}\right)=F,
$$

where $F$ is the external load. Using (4.3) and only first order terms, we have our desired linear equation

$$
\rho A_{c} \partial_{t}^{2} u-672 A_{c} \partial_{y}^{2} u(t, y)+\frac{256 A_{c}}{\tau} \int_{0}^{t} e^{-(t-s) / \tau} \partial_{y}^{2} u(s, y) d s=F
$$

\section{A nonlinear reptation model}

In the previous section the deformations of the PC-molecules and the CC-molecules were assumed to be related according to the formula

$$
\frac{\Delta \ell}{\Delta L}=\frac{\ell}{L}
$$

leading to the relationship

$$
\frac{\partial \lambda_{j p}}{\partial \lambda_{i c}}=\frac{\lambda_{j p}}{\lambda_{i c}} \delta_{i j}
$$

between the stretches.

The above relationship has the merit that it would make it feasible for the PC-molecule to 
remain within the constraining tube during relaxation. However, a more realistic and general relationship is of the form

$$
\frac{\Delta \ell}{\Delta L}=f\left(t, \frac{\ell}{L}\right), \quad \text { or } \quad \frac{\partial \lambda_{j p}}{\partial \lambda_{i c}}=f\left(t, \frac{\lambda_{j p}}{\lambda_{i c}}\right) \delta_{i j},
$$

where $f$ is a material dependent nonlinearity. With this more general relationship between the deformations, the relaxation process after an instantaneous step-strain in the time interval $0=t_{0}<t<t_{1}$ is given by

$$
\ell(t)=\ell_{0}+f\left(t_{0}, \frac{\ell_{0}}{L_{0}}\right) \Delta L_{0} e^{\frac{\left(t-t_{0}\right)}{\tau}} .
$$

In the time interval $\left(t_{m-1}, t_{m}\right), m \geq 1$ the relaxation process has the form

$$
\ell(t)=\ell_{0}+\sum_{i=0}^{m-1} f\left(t_{i}, \frac{\ell_{i}}{L_{i}}\right) \Delta L_{i} e^{\frac{-\left(t-t_{i}\right)}{\tau}} .
$$

In the limit, as $\Delta t=t_{i}-t_{i-1}$ tends to zero, we have

$$
\ell(t)=\int_{0}^{t} f\left(s, \frac{\ell(s)}{L(s)}\right) \frac{d L(s)}{d s} e^{-\frac{(t-s)}{\tau}} d s .
$$

In differential form we have

$$
\frac{d \ell}{d t}=f\left(t, \frac{\ell(t)}{L(t)}\right) \frac{d L}{d t}-\frac{1}{\tau}\left(\ell(t)-\ell_{0}\right) .
$$

We can write (5.2) in terms of the stretches of the PC-molecules $\lambda_{i p}, i=1,2,3$ and those of the CC-molecules $\lambda_{i c}, i=1,2,3$. When writing (5.2) in terms of the stretches, we take $\ell_{0}=1$. In particular, setting $\varepsilon_{1}=\delta u_{p}=\lambda_{1 p}-1$, we have from (5.2) the equation

$$
\dot{\varepsilon}_{1}+\frac{1}{\tau} \varepsilon_{1}=\dot{\lambda}_{1 c} f\left(t, \frac{1+\varepsilon_{1}}{\lambda_{1 c}}\right) .
$$

In the problem at hand $\lambda_{1 c}^{2} \lambda_{2 c}^{2}=1$, and from (3.2),

$$
\begin{aligned}
\frac{1}{\lambda_{1 c}}=\lambda_{2 c} & =1-\frac{1}{2} u^{\prime}(y)+\frac{1}{4} u^{\prime}(y)^{2}-\frac{1}{16} u^{\prime}(y)^{3} \pm \cdots \\
& =1-\varepsilon+\varepsilon^{2}-\frac{1}{2} \varepsilon^{3} \pm \cdots
\end{aligned}
$$

where we set $\varepsilon=\frac{1}{2} u^{\prime}(y)$. Next we write (5.3) as

$$
\begin{aligned}
\dot{\varepsilon}_{1}+\frac{1}{\tau} \varepsilon_{1}= & \dot{\varepsilon}\left(1+2 \varepsilon+\frac{3}{2} \varepsilon^{2}+\cdots\right) \\
& \cdot f\left(t, 1+\varepsilon_{1}-\varepsilon-\varepsilon_{1} \varepsilon+\varepsilon^{2}+\varepsilon_{1} \varepsilon^{2}-\frac{1}{2} \varepsilon^{3} \pm \cdots\right) .
\end{aligned}
$$


In the case $f$ is independent of $t$, which we assume henceforth, we expand $f$ about the point 1 and obtain

$$
\begin{aligned}
\dot{\varepsilon}_{1}+\frac{1}{\tau} \varepsilon_{1}= & \dot{\varepsilon}\left(1+2 \varepsilon+\frac{3}{2} \varepsilon^{2}+\cdots\right) \\
& \cdot\left\{f(1)+\dot{f}(1)\left(\varepsilon_{1}-\varepsilon-\varepsilon_{1} \varepsilon+\varepsilon^{2}+\varepsilon_{1} \varepsilon^{2}-\frac{1}{2} \varepsilon^{3}+-\cdots\right)+\cdots\right\} .
\end{aligned}
$$

In Section $3, f\left(t, \frac{\lambda_{1 p}}{\lambda_{1 c}}\right)=\frac{\lambda_{1 p}}{\lambda_{1 c}}$. In this case (5.4) becomes

$$
\begin{aligned}
\dot{\varepsilon}_{1}+\frac{1}{\tau} \varepsilon_{1}= & \dot{\varepsilon}\left(1+2 \varepsilon+\frac{3}{2} \varepsilon^{2}+\cdots\right) \\
& \cdot\left\{1+\varepsilon_{1}-\varepsilon-\varepsilon_{1} \varepsilon+\varepsilon^{2}+\varepsilon_{1} \varepsilon^{2}-\frac{1}{2} \varepsilon^{3} \pm \cdots+\cdots\right\} .
\end{aligned}
$$

We note that a special case of this latter equation is

$$
\begin{aligned}
\dot{\varepsilon}_{1}+\frac{1}{\tau} \varepsilon_{1} & =\dot{\varepsilon}\left(1+2 \varepsilon+\frac{3}{2} \varepsilon^{2}\right) \\
& =\frac{d}{d t}\left(\varepsilon+\varepsilon^{2}+\frac{1}{2} \varepsilon^{3}\right),
\end{aligned}
$$

which is itself a special case of the general model

$$
\dot{\varepsilon}_{1}+\frac{1}{\tau} \varepsilon_{1}=\frac{d}{d t}\left(b_{1} \varepsilon+b_{2} \varepsilon^{2}+b_{3} \varepsilon^{3}\right) .
$$

These, of course, are both special cases of the general model

$$
\dot{\varepsilon}_{1}+\frac{1}{\tau} \varepsilon_{1}=g_{v}(\varepsilon, \dot{\varepsilon}) .
$$

Neglecting all higher order terms in (5.5), we find

$$
\dot{\varepsilon}_{1}+\frac{1}{\tau} \varepsilon_{1}=\dot{\varepsilon}
$$

which is (3.10) of Section 4.

The dynamics of the rubber (see Section 4) are given by

$$
\rho A_{c} \partial_{t}^{2} u-\frac{\partial}{\partial y}\left(\frac{\partial W_{c c}}{\partial u_{y}}+\frac{\partial W_{p c}}{\partial u_{y}}\right)=F .
$$

Now

$$
\begin{aligned}
\frac{\partial W_{p c}}{\partial u_{y}} & =\frac{\partial W_{p c}}{\partial \lambda_{1 c}} \frac{\partial \lambda_{1 c}}{\partial u_{y}} \\
& =\frac{\partial W_{p c}}{\partial \lambda_{1 p}} \frac{\partial \lambda_{1 p}}{\partial \lambda_{1 c}} \frac{\partial \lambda_{1 c}}{\partial u_{y}} \\
& \approx \frac{\partial W_{p c}}{\partial \lambda_{1 p}} f\left(\frac{\lambda_{1 p}}{\lambda_{1 c}}\right) \frac{1}{2} \\
& =512 \varepsilon_{1} f\left(1+\varepsilon_{1}-\varepsilon-\varepsilon_{1} \varepsilon+\varepsilon^{2}+\varepsilon_{1} \varepsilon^{2}-\frac{1}{2} \varepsilon^{3} \pm \cdots\right)
\end{aligned}
$$


From (5.9) we have now

$$
\rho A_{c} \partial_{t}^{2} u-\partial_{y}\left\{g_{e}(\varepsilon)+512 \varepsilon_{1} f\left(1+\varepsilon_{1}-\varepsilon-\varepsilon_{1} \varepsilon+\varepsilon^{2}+\varepsilon_{1} \varepsilon^{2}-\frac{1}{2} \varepsilon^{3} \pm \cdots\right)\right\}=F,
$$

where $g_{e}(\varepsilon)=832 \varepsilon+a_{2} \varepsilon^{2}+\cdots$. This is a general nonlinear model based on the nonlinear constitutive relationship involving $f$ of (5.1) and that of (4.1). In Section 3 we had the simpler proportional assumption $f\left(\frac{\lambda_{1 p}}{\lambda_{1 c}}\right)=\frac{\lambda_{1 p}}{\lambda_{1 c}}$ as well as $g_{e}(\varepsilon) \approx 832 \varepsilon$. In that case $(5.10)$ becomes

$$
\rho A_{c} \partial_{t}^{2} u-\partial_{y}\left(832 \varepsilon+512 \varepsilon_{1}\left(1+\varepsilon_{1}-\varepsilon-\varepsilon_{1} \varepsilon+\varepsilon^{2}+\varepsilon_{1} \varepsilon^{2}-\frac{1}{2} \varepsilon^{3}+-\cdots\right)\right)=F .
$$

If we retain only first order terms in $\varepsilon, \varepsilon_{1}$ in (5.11) we obtain the equation

$$
\rho A_{c} \partial_{t}^{2} u-\partial_{y}\left(832 \varepsilon+512 \varepsilon_{1}\right)=F .
$$

Thus, a linearization of (5.11) and (5.5) gives

$$
\begin{aligned}
\rho A_{c} \partial_{t}^{2} u-\partial_{y}\left(832 \varepsilon+512 \varepsilon_{1}\right) & =F \\
\dot{\varepsilon}_{1}+\frac{1}{\tau} \varepsilon_{1} & =\dot{\varepsilon}
\end{aligned}
$$

which, recalling (3.10) and $\varepsilon=\frac{1}{2} u_{y}$, we see is the same as (4.4) of Section 4 .

\section{The nonlinear model and simple shear experiments}

Model development and identification processes (inverse problems with experimental data) for nonlinearities and parameters were carried out for filled elastomers undergoing simple shear deformation. A series of dynamic experiments were designed and carried out, each involving one or more "double sandwich" fixtures with layers of filled rubber at the interfaces as depicted in Figure 2.

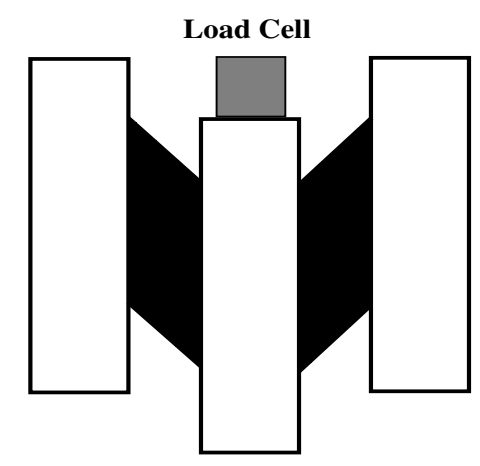

Figure 2: Schematic of the double lap simple shear experimental device. 
The side bars were fixed, while the middle bar was either perturbed by an impulsive hammer hit or provided with an initial strain and then released. In all experiments, both accelerometer and load cell data were collected for the motion of the middle bar. The samples consisted of A225 highly filled rubber with dimensions $0.1 \times 0.1 \times 0.8 \mathrm{in}$. with two such layers in each of the double lap devices as seen in Figure 2.

For simple shear, the model developed and used is based on (5.10) with Kelvin-Voigt damping added as given by

$$
\begin{aligned}
& \rho A_{c} u_{t t}-C_{D} u_{t y y}-\frac{\partial}{\partial y} A_{c} \sigma=0, \quad 0<y<\ell, \\
& \left\{M u_{t t}(t, \ell)+C_{D} u_{t y}(t, \ell)+A_{c} \sigma(t, \ell)\right\}=F(t)+M g, \\
& u(t, 0)=0, \\
& u(0, y)=u_{0} \quad u_{t}(0, y)=0 .
\end{aligned}
$$

Here $\rho$ is the mass density, $A_{c}$ is the cross sectional area of the sample, $C_{D}$ is a damping coefficient, $M$ is the mass of a tip body possibly attached to the middle bar ( $M=0$ if the tip mass is not included in the experimental set-up), $F(t)$ is an applied (at $y=\ell$ ) external force when present, and $\sigma$ is the elastic and viscoelastic shear stress given in general form by

$$
\begin{gathered}
\sigma\left(\varepsilon, \varepsilon_{1}\right)=g_{e}(\varepsilon)+\varepsilon_{1} \\
\dot{\varepsilon}_{1}+\frac{1}{\tau} \varepsilon_{1}=g_{v}(\varepsilon, \dot{\varepsilon}),
\end{gathered}
$$

as developed in the previous sections of this paper.

For each experimental data set, a least-squares minimization problem was carried out to identify the unknown parameters $q$ in the nonlinearities $g_{e}$ and $g_{v}$ using the accelerometer data $\left\{a_{i}\right\}$ (the load cell data was not used in the optimization procedure) with the corresponding model solutions $u_{t t}\left(t_{i}, \ell ; q\right)$ in

$$
J(q)=\frac{1}{2} \sum_{i=1}^{n}\left|a_{i}-u_{t t}\left(t_{i}, \ell ; q\right)\right|^{2} .
$$

The resulting optimized model was then used to generate load cell values which were then compared to the corresponding experimental load cell data.

Initial experiments involved one double-lap device with an attached tip mass (to produce deformations in shear sufficient for observation) which was excited with a hammer hit on the middle bar. The experiment was performed with highly filled rubber samples with different amounts of attached extra mass. In general, we found that the data collected with no extra mass on the sample could be approximated well with the above type models even when the viscoelastic response function $g_{v}$ is assumed to be linear. This is not surprising, since the maximum strain levels in these type of experiments were below $10 \%$. However, when extra mass was involved to achieve larger deformations and strain levels (this required huge masses!), the experiment did not provide suitable data, since additional "tilting" modes became excited and these could not be accounted for by the above one-dimensional model. We subsequently redesigned the experimental device to consist of a hub with four identical 
arms, each containing a double lap device as pictured in Figure 2. The arms could be latched down so as to produce initial strains of up to $100 \%$. Upon quick release, this device produced very clean free-release data for the samples undergoing simple shear with no additional deformations; these proved adequate for our model estimation and validation. Thus, the experiments consisted of latching down the four arms of the fixture at some prescribed initial strain $(50 \%, 70 \%$ and $100 \%$ strain) and then suddenly releasing the latch. We began with approximately $50 \%$ initial shear strain and repeated each release experiment three times. We again collected both accelerometer and load cell data. The sequence of experiments was repeated with about $70 \%$ and $100 \%$ initial shear strains, respectively.

For our model fitting we used a number of different constitutive relationships between the stress and the strain, including linear and nonlinear functions for both $g_{e}$ and $g_{v}$. The identification problem was first performed for the data obtained for $100 \%$ initial strain. We quickly found that a linear viscoelastic response function $g_{v}$ was no longer adequate to describe the data in this higher strain regime. Thus, we were led to models of the form

$$
\sigma=\tilde{g}_{e}\left(\frac{\partial u}{\partial y}\right)+C_{D} \frac{\partial^{2} u}{\partial t \partial y}+\varepsilon_{1}, \quad \dot{\varepsilon}_{1}+\frac{1}{\tau} \varepsilon_{1}=\tilde{g}_{v}\left(\frac{\partial u}{\partial y}, \frac{\partial^{2} u}{\partial t \partial y}\right),
$$

where $\tilde{g}_{e}$ and $\tilde{g}_{v}$ are cubic polynomials and $\tilde{g}_{v}$ may or may not depend on $\frac{d \varepsilon}{d t}=\frac{1}{2} \frac{\partial^{2} u}{\partial t \partial y}$, (i.e., $\tilde{g}_{v i}=\tilde{g}_{v d}$ if the nonlinearities are the same in both increasing and decreasing deformation). The notion that one might need $\tilde{g}_{v}{ }^{\prime} s$ of the form

$$
\tilde{g}_{v}\left(u_{y}, \dot{u}_{y}\right)= \begin{cases}\tilde{g}_{v i}\left(u_{y}\right) & \text { if } \dot{u}_{y} \geq 0,\left(u_{y} \text { increasing }\right) \\ \tilde{g}_{v d}\left(u_{y}\right) & \text { if } \dot{u}_{y}<0,\left(u_{y} \text { decreasing }\right)\end{cases}
$$

was motivated by earlier results $[4,6,7]$ with similar materials undergoing tensile deformations where such models were necessary because stress-strain curves clearly indicated that the nonlinearities are distinctly different for contraction versus elongation.

The parameters in a linear $\tilde{g}_{v i}=\tilde{g}_{v d}$ that provided the best fit for the $100 \%$ initial strain data (see Figure 3) did not produce a model that described well the $70 \%$ and $50 \%$ initial strain experiments. Thus we next used the full nonlinear hysteretic model (i.e., $\tilde{g}_{v i} \neq \tilde{g}_{v d}$ in (6.8) taken as cubic nonlinearities) to approximate the $100 \%$ initial strain data and found several different sets of cubic nonlinearities that gave a very good approximation. Again, earlier efforts with these materials suggested that cubic (as opposed to higher order) nonlinearities might be sufficient to capture the dynamics. To find a set that also provides a good fit for the $70 \%$ initial data we optimized on both data sets simultaneously (i.e., we used both data sets in the cost criterion (6.7)). The best fit in this case is depicted in Figures 4 and 5 . We found that the set of parameters identified in this case described both data sets with very good accuracy (Figures 4,5). Finally, we found that the $50 \%$ initial strain data sets were the most difficult to use with the models (see Figure 6). Our investigations suggest that in this data, the deformations are likely too small to obtain reliable fits due to unfavorable signal to noise ratios. 

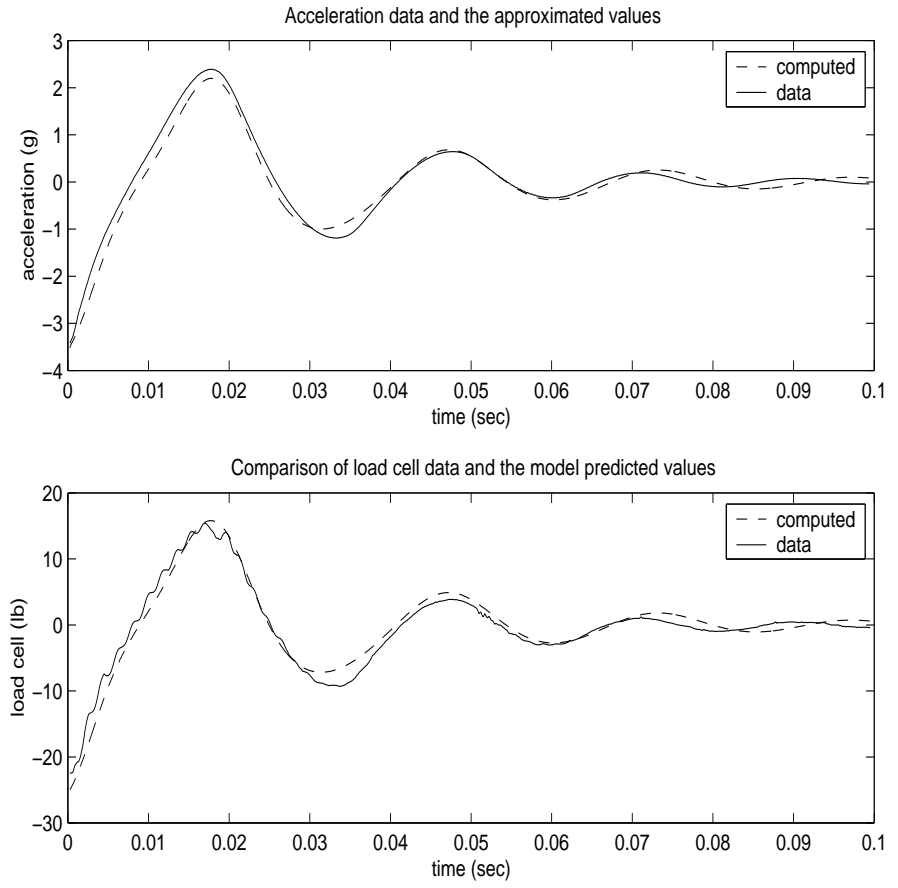

Figure 3: Hysteretic model with $\tilde{g}_{v i}=\tilde{g}_{v d}$ linear for $100 \%$ initial shear strain.
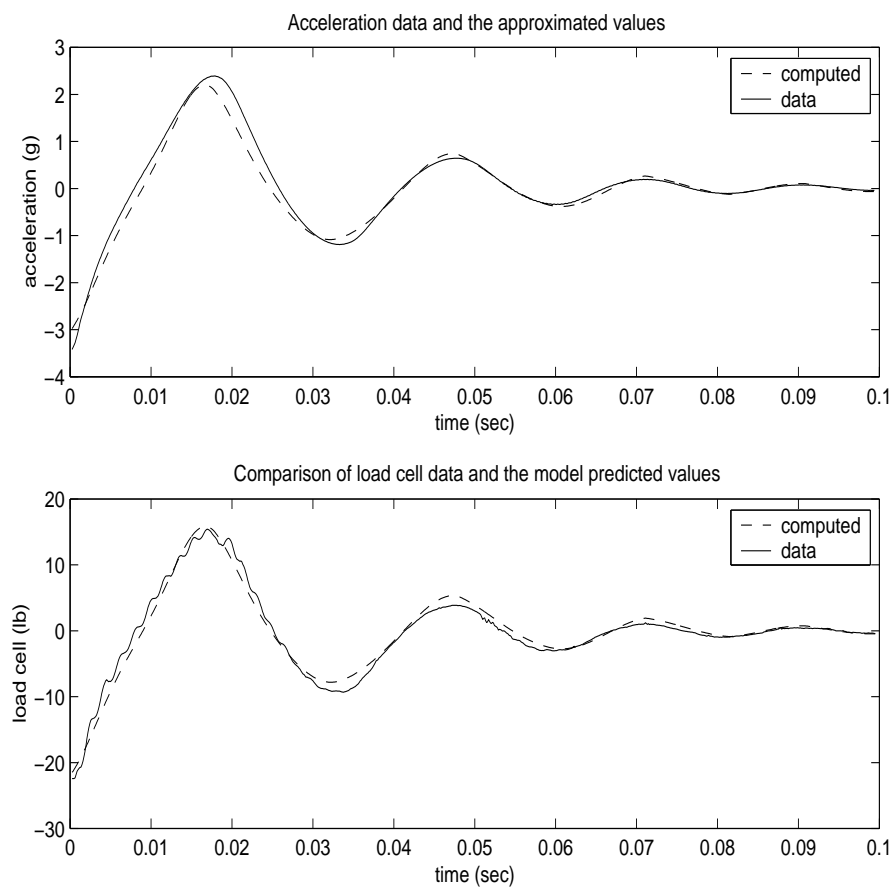

Figure 4: Model with $\tilde{g}_{v i} \neq \tilde{g}_{v d}$ cubic best fit compared with $100 \%$ initial shear strain data response. 

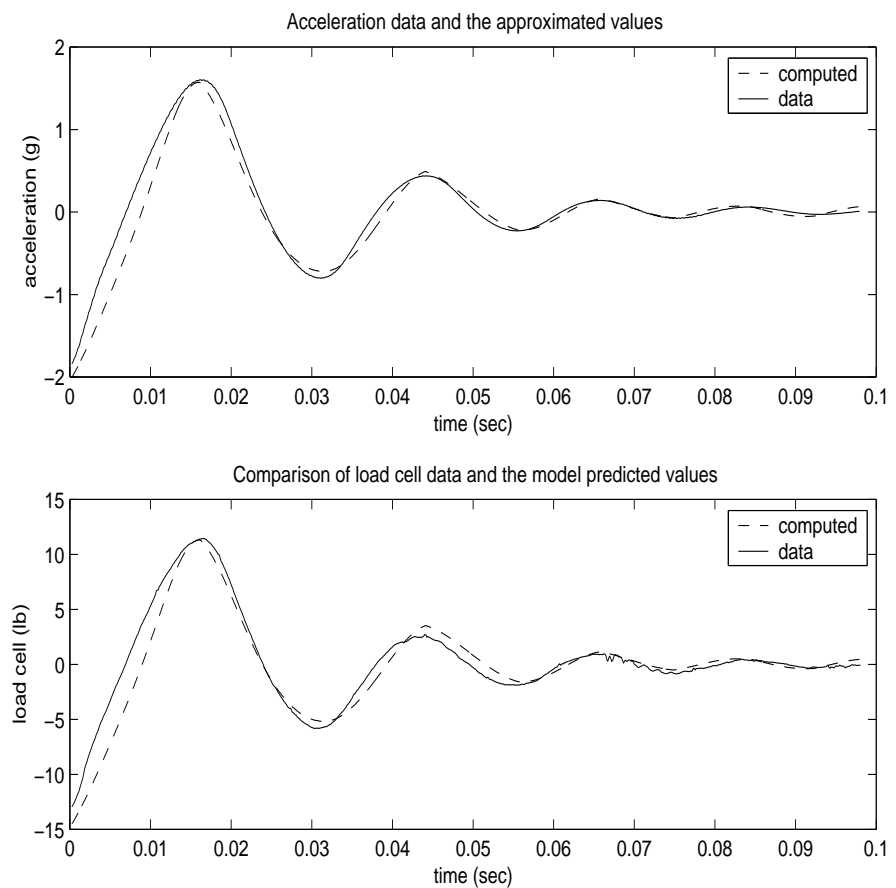

Figure 5: Model with the same (as in Figure 4) $\tilde{g}_{v i} \neq \tilde{g}_{v d}$ cubic best fit compared with $70 \%$ initial shear strain data response.
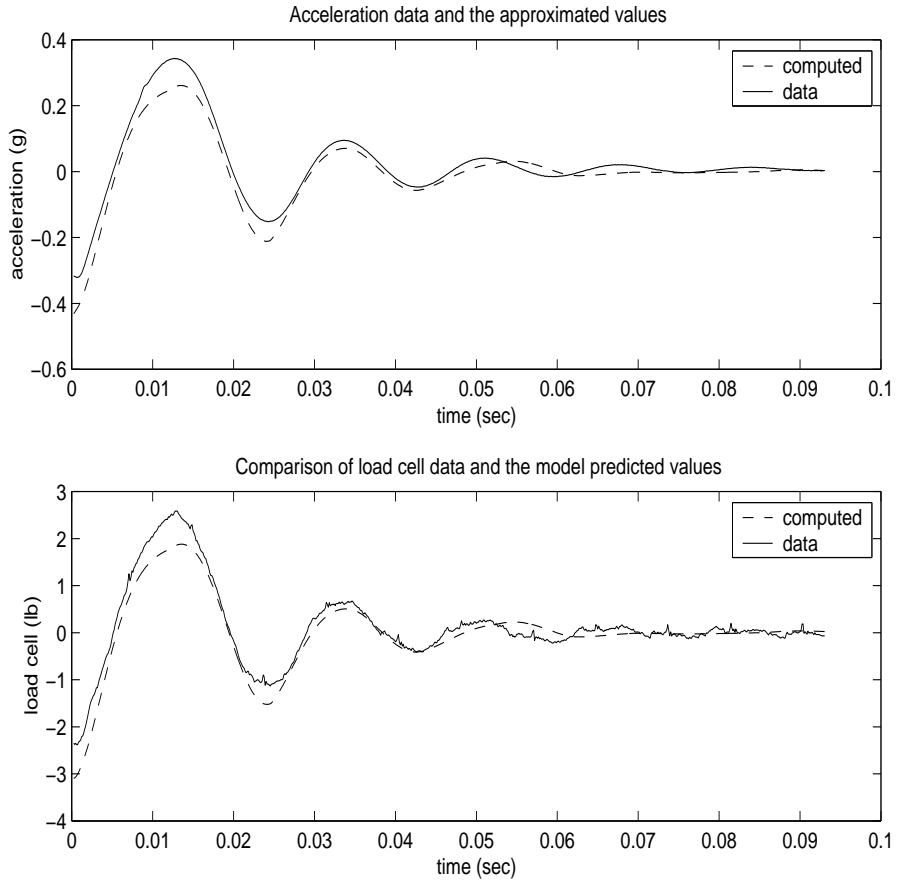

Figure 6: Best model with $\tilde{g}_{v i} \neq \tilde{g}_{v d}$ cubic compared with $50 \%$ initial shear strain data response. 


\section{Analysis of the linear model equation}

In this section we analyze the linear model equation (4.4) and make some conclusion about the model. Dividing by $\rho A_{c}$ in (4.4) we obtain the equation

$$
\partial_{t}^{2} u-\frac{672}{\rho} \partial_{y}^{2} u+\frac{256}{\rho \tau} \int_{0}^{t} e^{-(t-s) / \tau} \partial_{y}^{2} u(s, y) d s=\frac{1}{\rho A_{c}} F .
$$

We will later on solve (7.1) with boundary and initial conditions given by

$$
u(t, 0)=0, \quad \frac{\partial u}{\partial y}(t, b)=G(t), \quad u(0, y)=\partial_{t} u(0, y)=0 .
$$

To facilitate our analysis, we set

$$
v=\frac{672}{\rho} u-\frac{256}{\rho \tau} \int_{0}^{t} e^{-(t-s) / \tau} u(s, y) d s .
$$

Then from (7.1) we obtain

$$
\begin{aligned}
v_{t t}+\frac{256}{672 \tau} v_{t}-\frac{416}{672 \tau^{2}} v-\frac{672}{\rho} v_{y y} & +\left(\frac{416}{672 \tau}\right)^{2} \int_{0}^{t} e^{-\frac{416}{672 \tau}(t-s)}\left(v_{s}+\frac{1}{\tau} v\right) d s \\
& =\frac{672}{\rho^{2} A_{c}} F-\frac{672}{\rho}\left(\frac{416}{672 \tau}\right)^{2} e^{-\frac{416}{672 \tau} t} u(0, y)
\end{aligned}
$$

We rewrite the initial and boundary conditions in (7.2), using (7.3), as follows:

$$
\begin{gathered}
v(t, 0)=0 \\
\frac{\rho}{464} \int_{0}^{t} e^{-\frac{416}{672 \tau}(t-s)}\left[v_{s y}(s, b)+\frac{1}{\tau} v_{y}(s, b)\right] d s+\partial_{y} u(0, b) e^{-\frac{416}{672 \tau} t}=G(t) \\
v(0, y)=0 \\
v_{t}(0, y)+\frac{1}{\tau} v(0, y)=0 .
\end{gathered}
$$

The second equation in (7.5) can be written as

$$
\begin{array}{r}
\frac{\rho}{672}\left[v_{y}(t, b)-e^{-\frac{416}{672 \tau} t} v_{y}(0, b)\right]+\frac{256 \rho}{(672)^{2} \tau} \int_{0}^{t} e^{-\frac{416}{672 \tau}(t-s)} v_{y}(s, b) d s+G\left(0^{+}\right) e^{-\frac{416}{672 \tau} t} \\
=G(t) .
\end{array}
$$

We next consider the Sturm-Liouville problem

$$
\begin{aligned}
-Z^{\prime \prime}-\lambda Z & =0 \\
Z(a)-h_{0} Z^{\prime}(a) & =0, \quad h_{0} \geq 0 \\
Z(b)-h_{1} Z^{\prime}(b) & =0, \quad h_{1} \geq 0 .
\end{aligned}
$$

We recall that there is a corresponding sequence of eigenvalues $0<\lambda_{1}<\lambda_{2}<\ldots<\lambda_{n}<$ $\ldots \nearrow \infty$ and corresponding orthonormal eigenfunctions $\varphi_{1}, \varphi_{2}, \ldots, \varphi_{n}, \ldots$ 
Consider a solution of (7.4)-(7.6) in the form

$$
v(t, y)=\sum_{n=1}^{\infty} v_{n}(t) \varphi_{n}(y)
$$

Writing

$$
f(t, y)=\sum_{n=1}^{\infty} f_{n}(t) \varphi_{n}(y)
$$

where

$$
f(t, y)=\frac{672}{\rho^{2} A_{c}} F-\frac{672}{\rho}\left(\frac{416}{672 \tau}\right)^{2} e^{-\frac{416}{672 \tau} t} u(0, y),
$$

we have from (7.4) the equation

$$
\begin{gathered}
v_{n}^{\prime \prime}(t)+\frac{256}{672 \tau} v_{n}^{\prime}+\left(\frac{672}{\rho} \lambda_{n}-\frac{416}{672 \tau^{2}}\right) v_{n} \\
+\left(\frac{416}{672 \tau}\right)^{2} \int_{0}^{t} e^{-\frac{416}{672 \tau}(t-s)}\left(v_{n}^{\prime}(s)+\frac{1}{\tau} v_{n}(s)\right) d s=f_{n}(t)
\end{gathered}
$$

Set

$$
w_{n}(t)=\left(\frac{416}{672 \tau}\right)^{2} \int_{0}^{t} e^{-\frac{416}{672 \tau}(t-s)}\left(v_{n}^{\prime}(s)+\frac{1}{\tau} v_{n}(s)\right) d s
$$

Then

$$
w_{n}^{\prime}(t)=\left(\frac{416}{672 \tau}\right)^{2}\left(v_{n}^{\prime}(t)+\frac{1}{\tau} v_{n}(t)\right)-\frac{416}{672 \tau} w_{n}(t) .
$$

Let

$$
\begin{aligned}
u_{1}^{n}(t) & =v_{n}(t) \\
u_{2}^{n}(t) & =v_{n}^{\prime}(t) \\
u_{3}^{n}(t) & =w_{n}(t) \\
d & =\frac{416}{672 \tau} \\
\Delta_{n} & =\frac{672}{\rho} \lambda_{n}-\frac{416}{672 \tau^{2}} .
\end{aligned}
$$

From (7.7) we have

$$
\frac{d}{d t}\left(\begin{array}{c}
u_{1}^{n} \\
u_{2}^{n} \\
u_{3}^{n}
\end{array}\right)=\left(\begin{array}{ccc}
0 & 1 & 0 \\
-\Delta_{n} & \frac{-256}{672 \tau} & -1 \\
\frac{d^{2}}{\tau} & d^{2} & -d
\end{array}\right)\left(\begin{array}{c}
u_{1}^{n} \\
u_{2}^{n} \\
u_{3}^{n}
\end{array}\right)+\left(\begin{array}{c}
0 \\
f_{n} \\
0
\end{array}\right)
$$


Next define

$$
A_{n}=\left(\begin{array}{ccc}
0 & 1 & 0 \\
-\Delta_{n} & \frac{-256}{672 \tau} & -1 \\
\frac{d^{2}}{\tau} & d^{2} & -d
\end{array}\right)
$$

Then

$$
\left|\xi I-A_{n}\right|=\xi^{3}+\left(\frac{256}{672 \tau}+d\right) \xi^{2}+\left(d^{2}+\frac{256}{672 \tau} d+\Delta_{n}\right) \xi+d \Delta_{n}+\frac{d^{2}}{\tau} .
$$

The Routh-Hurwitz Theorem guarantees that the roots of the polynomial equation

$$
z^{3}+a_{1} z^{2}+a_{2} z+a_{3}=0
$$

have negative real parts if the following conditions are met:

(i) $a_{1}>0$

(ii)

$$
\left|\begin{array}{cc}
a_{1} & a_{3} \\
1 & a_{2}
\end{array}\right|>0
$$

(iii) $a_{3}>0$.

Thus the roots of $\left|\xi I-A_{n}\right|=0$ have negative real parts if

$$
\left|\begin{array}{cc}
\frac{256}{672 \tau}+d & \Delta_{n} d+\frac{d^{2}}{\tau} \\
1 & \frac{256}{672 \tau} d+d^{2}+\Delta_{n}
\end{array}\right|>0
$$

Thus, the eigenvalues of $A_{n}$ have negative real parts if

$$
d\left(\frac{256}{672 \tau}+d\right)^{2}+\left(\frac{256}{672 \tau}+d\right) \Delta_{n}-\Delta_{n} d-\frac{d^{2}}{\tau}>0
$$

Now recalling that

$$
\begin{aligned}
d & =\frac{416}{672 \tau} \\
\Delta_{n} & =\frac{672}{\rho} \lambda_{n}-\frac{416}{672 \tau^{2}},
\end{aligned}
$$

a simple calculation reveals that the inequality in (7.10) is true if $\lambda_{n}>0$. Thus the eigenvalues of $A_{n}$ have negative real parts.

From (7.8), (7.9) we have

$$
\left(\begin{array}{c}
v_{n}(t) \\
v_{n}^{\prime}(t) \\
w_{n}(t)
\end{array}\right)=e^{t A_{n}}\left(\begin{array}{c}
v_{n}(0) \\
v_{n}^{\prime}(0) \\
0
\end{array}\right)+\int_{0}^{t} e^{(t-s) A_{n}}\left(\begin{array}{c}
0 \\
f_{n}(s) \\
0
\end{array}\right) d s
$$

Remark 7.1: Since the eigenvalues of $A_{n}$ have real parts negative we have

$$
e^{t A_{n}}\left(\begin{array}{c}
v_{n}(0) \\
v_{n}^{\prime}(0) \\
0
\end{array}\right) \longrightarrow\left(\begin{array}{l}
0 \\
0 \\
0
\end{array}\right), \quad \text { as } t \rightarrow \infty
$$




\section{Solution of the linearized model equation}

In this section we proceed to solve (7.4), (7.5). For convenience we rewrite (7.4) and (7.5) as follows:

$$
\begin{aligned}
v_{t t}+\frac{256}{672 \tau} v_{t}-\frac{416}{672 \tau^{2}} v-\frac{672}{\rho} v_{y y} & +\left(\frac{416}{672 \tau}\right)^{2} \int_{0}^{t} e^{-\frac{416}{672 \tau}(t-s)}\left(v_{s}+\frac{1}{\tau} v\right) d s \\
& =\frac{672}{\rho^{2} A_{c}} F-\frac{672}{\rho}\left(\frac{416}{672 \tau}\right)^{2} e^{-\frac{416}{672 \tau} t} u(0, y) .
\end{aligned}
$$

The initial and boundary conditions are

$$
\begin{array}{r}
v(0, y)=0, \\
v_{t}(0, y)=0, \\
v(t, 0)=0,
\end{array}
$$

and

$$
\begin{array}{r}
\frac{\rho}{672}\left[v_{y}(t, b)-e^{-\frac{416}{672} t} v_{y}(0, b)\right]+\frac{256 \rho}{(672)^{2} \tau} \int_{0}^{t} e^{-\frac{416}{672 \tau}(t-s)} v_{y}(s, b) d s \\
+G\left(0^{+}\right) e^{-\frac{416}{672 \tau} t}=G(t) .
\end{array}
$$

Set

$$
\tilde{w}=-\frac{\rho}{672} e^{-\frac{416}{672 \tau} t} v_{y}(0, b)+\frac{256 \rho}{(672)^{2} \tau} \int_{0}^{t} e^{-\frac{416}{672 \tau}(t-s)} v_{y}(s, b) d s .
$$

Then equation (8.3) becomes

$$
\frac{\rho}{672} v_{y}(t, b)+\tilde{w}+G\left(0^{+}\right) e^{-\frac{416}{672 \tau} t}=G(t)
$$

Differentiating (8.3) we have

$$
\frac{\rho}{672} \frac{d}{d t} v_{y}(t, b)-\frac{416}{672 \tau} \tilde{w}+\frac{256 \rho}{(672)^{2} \tau} v_{y}(t, b)-\frac{416}{672 \tau} G\left(0^{+}\right) e^{-\frac{416}{672 \tau} t}=G^{\prime}(t),
$$

where we have assumed that $G$ is sufficiently regular, e.g., $G \in C^{1}$. From (8.5) and (8.6) we have

$$
\frac{\rho}{672} \frac{d}{d t} v_{y}(t, b)+\frac{\rho}{672 \tau} v_{y}(t, b)=G^{\prime}(t)+\frac{416}{672 \tau} G(t)
$$

and from (8.2) we see that

$$
v_{y}(0, b)=0 .
$$

From (8.7), and (8.8) we thus see that we can give $v_{y}(t, b)$ explicitly in terms of the function $G$. Thus we write

$$
v_{y}(t, b)=\eta(t)
$$


where $\eta$ can be explicitly determined in terms of the function $G$. Thus, we replace (8.2) and (8.3) by

$$
\begin{aligned}
v(0, y) & =v_{t}(0, y)=0 \\
v(t, 0) & =0 \\
v_{y}(t, b) & =\eta(t) .
\end{aligned}
$$

We now proceed to solve (8.1) with initial and boundary conditions given by (8.9). Toward this goal let

$$
Q(t, y)=v(t, y)-y \eta(t)
$$

In terms of $Q$ the equation (8.1) becomes

$$
Q_{t t}+\frac{256}{672 \tau} Q_{t}-\frac{416}{672 \tau^{2}} Q-\frac{672}{\rho} Q_{y y}+\left(\frac{416}{672 \tau}\right)^{2} \int_{0}^{t} e^{-\frac{416}{672 \tau}(t-s)}\left(Q_{s}+\frac{1}{\tau} Q\right) d s=\tilde{f}
$$

where

$$
\tilde{f}=f-y \eta^{\prime \prime}(t)-\frac{256}{672 \tau} y \eta^{\prime}(t)+\frac{416}{672 \tau^{2}} y \eta(t)-\left(\frac{416}{672 \tau}\right)^{2} \int_{0}^{t} e^{-\frac{416}{672 \tau}(t-s)}\left\{y \eta^{\prime}(s)+\frac{1}{\tau} y \eta(s)\right\} d s .
$$

For initial and boundary conditions we have

$$
\begin{aligned}
Q(0, y) & =-y \eta(0) \\
Q_{t}(0, y) & =-y \eta^{\prime}(0) \\
Q(t, 0) & =Q_{y}(t, b)=0 .
\end{aligned}
$$

Let us now consider the Sturm-Liouville problem

$$
\begin{aligned}
-Z^{\prime \prime}-\lambda Z & =0 \\
Z(0) & =0 \\
Z^{\prime}(b) & =0 .
\end{aligned}
$$

The eigenvalue problem (8.12) has a system of eigenvalues $\mu_{1}<\mu_{2}<\ldots \nearrow \infty$ and a corresponding orthonormal system of eigenfunctions $\psi_{1}, \psi_{2}, \ldots, \psi_{n}, \ldots$.. Let

$$
R(t, y)=\left(\frac{416}{672 \tau}\right)^{2} \int_{0}^{t} e^{-\frac{416}{672 \tau}(t-s)}\left\{Q_{s}+\frac{1}{\tau} Q\right\} d s
$$

We now write

$$
\begin{aligned}
R(t, y) & =\sum_{n=1}^{\infty} r_{n}(t) \psi_{n}(y), \\
\tilde{f}(t, y) & =\sum_{n=1}^{\infty} \tilde{f}_{n}(t) \psi_{n}(y), \\
Q(t, y) & =\sum_{n=1}^{\infty} q_{n}(t) \psi_{n}(y),
\end{aligned}
$$


and seek a solution for (8.10) and (8.11). Let

$$
\tilde{A}_{n}=\left(\begin{array}{ccc}
0 & 1 & 0 \\
-\tilde{\Delta}_{n} & \frac{-256}{672 \tau} & -1 \\
\frac{d^{2}}{\tau^{2}} & d^{2} & -d
\end{array}\right),
$$

where

$$
\tilde{\Delta}_{n}=\frac{672}{\rho} \mu_{n}-\frac{416}{672 \tau^{2}}
$$

Then

$$
\left(\begin{array}{c}
q_{n}(t) \\
q_{n}^{\prime}(t) \\
r_{n}(t)
\end{array}\right)=e^{t \tilde{A}_{n}}\left(\begin{array}{c}
q_{n}(0) \\
q_{n}^{\prime}(0) \\
0
\end{array}\right)+\int_{0}^{t} e^{(t-s) \tilde{A}_{n}}\left(\begin{array}{c}
0 \\
\tilde{f}_{n}(s) \\
0
\end{array}\right) d s
$$

where

$$
\begin{aligned}
& q_{n}(0)=-\eta(0) \int_{0}^{b} y \psi_{n}(y) d y \\
& q_{n}^{\prime}(0)=-\eta^{\prime}(0) \int_{0}^{b} y \psi_{n}(y) d y .
\end{aligned}
$$

Once $Q$ is determined the solution $v$ for (7.4) and (7.5) is given by

$$
v(t, y)=Q(t, y)+y \eta(t)
$$

Remark 8.1 The eigenvalues of $\tilde{A}_{n}$ have negative real parts if $\mu_{n}>0$. This is established using the Routh-Hurwitz theorem as was done earlier.

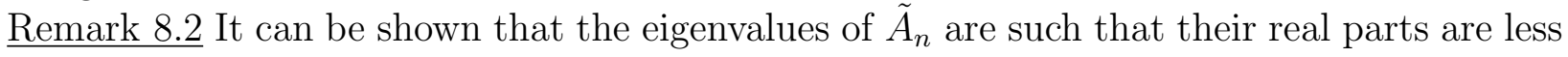
than a fixed negative real number for all $n$.

$\underline{\text { Remark 8.3 }}$ Because all the eigenvalues of $\tilde{A}_{n}$ have real parts negative, we have

$$
e^{t \tilde{A}_{n}}\left(\begin{array}{c}
q_{n}(0) \\
q_{n}^{\prime}(0) \\
0
\end{array}\right) \rightarrow\left(\begin{array}{l}
0 \\
0 \\
0
\end{array}\right), \quad \text { as } t \rightarrow \infty
$$

\section{Concluding remarks}

The stress strain models of (6.5)-(6.6) given by

$$
\begin{gathered}
\sigma\left(\varepsilon, \varepsilon_{1}\right)=g_{e}(\varepsilon)+\varepsilon_{1} \\
\dot{\varepsilon}_{1}+\frac{1}{\tau} \varepsilon_{1}=g_{v}(\varepsilon, \dot{\varepsilon}),
\end{gathered}
$$

discussed in Section 6 are special cases of more general models in which one admits heterogeneity in the PC-molecules. Specifically, one might have a distribution of different classes 
of PC-molecules, each characterized by different internal strains $\varepsilon_{i n}$ depending on physical properties such as length, relaxation time, etc. For example, the PC-molecules might have differing relaxation times $\tau$ so that $\varepsilon_{i n}=\varepsilon_{i n}(t, y ; \tau)$. For a continuum one would then have a probability distribution $P(\tau)$ on $\tau \in \mathcal{T}$, where $\mathcal{T}$ is the set of possible relaxation times. The corresponding stress strain models are then given by

$$
\sigma(t, y ; P)=g_{e}(\varepsilon(t, y), \dot{\varepsilon}(t, y))+\gamma \int_{\mathcal{T}} \varepsilon_{i n}(t, y ; \tau) d P(\tau)
$$

where $\varepsilon_{i n}(t, y ; \tau)$ satisfies, for each $\tau \in \mathcal{T}$,

$$
\dot{\varepsilon_{i n}}(t, y ; \tau)+\frac{1}{\tau} \varepsilon_{i n}(t, y ; \tau)=g_{v}(\varepsilon(t, y), \dot{\varepsilon}(t, y)) .
$$

The models we used with shear data in Section 6 required only a simple Dirac delta measure $d P(\tau)=\delta_{\tau_{1}}(\tau) d \tau$ with $\varepsilon_{\text {in }}=\varepsilon_{1}$ then satisfying

$$
\dot{\varepsilon}_{1}+\frac{1}{\tau_{1}} \varepsilon_{1}=g_{v}(\varepsilon, \dot{\varepsilon})
$$

in order to obtain reasonable fits to the experimental data. We compare this with the situation for tensile deformations reported in $[4,5]$ where the data required multiple relaxation times in the model so that the measure $P$ had atoms at $\tau_{1}$ and $\tau_{2}$, (i.e., the measure is composed of Dirac measures concentrated at $\tau_{1}$ and $\tau_{2}$ ), and the constitutive law led to the model

$$
\begin{aligned}
\sigma(t, y ; P) & =g_{e}(\varepsilon(t, y), \dot{\varepsilon}(t, y))+\gamma_{1} \varepsilon_{1}\left(t, y ; \tau_{1}\right)+\gamma_{2} \varepsilon_{2}\left(t, y ; \tau_{2}\right) \\
\dot{\varepsilon}_{1} & +\frac{1}{\tau_{1}} \varepsilon_{1}=\frac{d}{d t} g_{v}(\varepsilon, \dot{\varepsilon}) \\
\dot{\varepsilon}_{2} & +\frac{1}{\tau_{2}} \varepsilon_{2}=\frac{d}{d t} g_{v}(\varepsilon, \dot{\varepsilon}) .
\end{aligned}
$$

In the shear studies, we found that the nonlinear models (specifically, $g_{v}$ ) were essential in describing our data, but a simple uniform relaxation time was adequate for the PC-molecules.

\section{Acknowledgements}

This research was supported in part by the U.S. Air Force Office of Scientific Research under grants AFOSR-FA9550-04-1-0220 (HTB and NGM) and AFOSR F49620-03-1-0185(GAP). The authors would also like to express their sincere appreciation to Mike Gaitens of the Lord Corporation for his collaborative efforts on the simple shear experiments discussed in this paper.

\section{References}

[1] H.T. Banks and N.J. Lybeck, Modeling methodology for elastomer dynamics, Tech. Rep. CRSC-TR96-29, NCSU, 1996; in Systems and Control in the Twenty-First Century, (C. Byrnes, et al.,eds.), Birkhauser, PSCT22, 1996, 37-50. 
[2] H.T. Banks, N.J. Lybeck, M.J. Gaitens, B.C. Munoz, and L.C. Yanyo, Modeling the dynamic mechanical behaviour of elastomers, Tech.Rep. CRSC-TR96-26, NCSU, 1996.

[3] H.T. Banks and N.G. Medhin, A molecular based dynamic model for viscoelastic responses of rubber in tensile deformations, Communications on Applied Nonlinear Analysis, 8 (2001), 1-18.

[4] H.T. Banks, N.G. Medhin and G.A. Pinter, Multiscale considerations in modeling of nonlinear elastomers, CRSC-TR03-42, October, 2003; J. Comp. Meth. Sci. and Engr., to appear.

[5] H.T. Banks, N.G. Medhin and G.A. Pinter, Nonlinear reptation in molecular based hysteresis models for polymers, Quarterly Applied Math., 62 (2004), 767-779.

[6] H.T. Banks, G.A. Pinter, L.K. Potter, M.J. Gaitens and L.C. Yanyo, Modeling of nonlinear hysteresis in elastomers under uniaxial tension, J. Intelligent Material Systems and Structures 10, (1999), 116-134.

[7] H.T. Banks, G.A. Pinter, L.K. Potter, M.J. Gaitens and L.C. Yanyo, Models for nonlinear elastomers, Tech.Rep. CRSC-TR01-06, NCSU, 2001; Proceedings of the 2nd European Conference on Structural Control, July 3-6, 2000, Ecole National des Ponts et Chaussess, Champs-sur-Marne, France.

[8] M. Doi and S.F. Edwards, The Theory of Polymer Dynamics, Oxford, New York, 1986.

[9] J.D. Ferry, Viscoelastic Properties of Polymers, John Wiley and Sons, Inc., New York, 1961.

[10] A.R. Johnson, C.J. Quigley, D.G. Young and J.A. Danik, Viscohyperelastic modeling of rubber vulcanization, Tire Sci. Technology, 21 (1993), 179-199.

[11] A.R. Johnson and R.G. Stacer, Rubber viscoelasticity using the physically constrained system's stretches as internal variables, Rubber Chemistry and Technology, 66 (1993), $567-577$.

[12] R.W. Ogden, Non-Linear Elastic Deformations, Ellis Horwood Limited, Chichester, 1984.

[13] L.H. Sperling, Introduction to Physical Polymer Science, John Wiley \& Sons, 1992.

[14] L.R.G. Treloar, The Physics of Rubber Elasticity, Clarendon, Oxford 1975.

[15] K.C. Valanis and R.F. Landel, The strain-energy function of a hyperelastic material in terms of the extension ratios, J. Applied Physics, 38 (1967), 2997-3002. 